\title{
Linear Waves in Force-Free Fibrils
}

\author{
Y.D. Zhugzhda
}

Kiepenheuer-Institut für Sonnenphysik, Schöneckstr 6, D-79104

Freiburg, Germany

E-mail: yuzef@kis.uni-freiburg.de

\begin{abstract}
The advanced thin flux tube approximation for force-free thin magnetic flux tubes is used to derive a dispersion relation for linear waves. All wave modes appear to be coupled in a twisted flux tube. In the case of a weakly twisted flux tube, it has been found that torsional Alfvén waves have dispersion and produce pressure and temperature fluctuations. The effect of tube rotation is pointed out. These properties of linear waves have an impact on prominence oscillations.
\end{abstract}

\section{Introduction}

Theoretical treatments of oscillations of solar quiescent prominences are based on the well-developed theory of linear waves in current-free flux tubes and magnetic sheets. But the magnetic field of the prominences is not likely potential, because the helicity of filaments is observed. Thus, there is a need to examine the models of force-free magnetic filaments. The theory of linear waves in force-free flux tubes has not been developed up to the present time. The advanced thin flux tube approximation for force-free magnetic flux tubes, derived by Zhugzhda (1996) made it possible to obtain the dispersion equation for torsional, fast and slow body waves in twisted flux tubes. The distinctions of the linear waves in twisted and untwisted magnetic filaments are outlined. The effects of the twisting on the oscillations of the prominence filaments are discussed.

\section{Basic Equations}

The governing set of equations is the thin tube approximation for a straight, vertical, axisymmetric, force-free magnetic flux tube, which allows us to consider axisymmetric perturbations of the tube, and has been derived by Zhugzhda (1996). The dependent variables of the problem are the longitudinal component of the plasma velocity, $u$, the longitudinal component of the magnetic field, $B$, plasma density, $\rho$, and pressure, $p$, tube cross section, $A=\pi R^{2}$, $\phi$-components of the magnetic field, $B_{\phi}=J r$ and velocity, $v_{\phi}=\Omega r$, and the radial component of velocity, $v_{r}=v r$, where the radius coordinate, $r$, varies within the tube, $0 \leq r \leq R$. The set of the governing equations in these variables is

$$
\rho\left(\frac{\partial u}{\partial t}+u \frac{\partial u}{\partial z}\right)+\frac{\partial p}{\partial z}=0
$$




$$
\begin{gathered}
\frac{\partial}{\partial t}\left(\frac{\rho}{B}\right)+\frac{\partial}{\partial z}\left(u \frac{\rho}{B}\right)=0 \\
\frac{\partial}{\partial t}\left(\frac{\Omega}{B}\right)+u \frac{\partial}{\partial z}\left(\frac{\Omega}{B}\right)=\frac{B}{4 \pi \rho} \frac{\partial}{\partial z}\left(\frac{J}{B}\right) \\
\frac{\partial}{\partial t}\left(\frac{J}{B}\right)+\frac{\partial}{\partial z}\left(u \frac{J}{B}\right)=\frac{\partial \Omega}{\partial z} \\
\frac{d}{d t}\left(\frac{p}{\rho^{\gamma}}\right)=0 \\
\frac{\partial A}{\partial t}+u \frac{\partial A}{\partial z}-2 A v=0 \\
\frac{\partial B}{\partial t}+u \frac{\partial B}{\partial z}+2 B v=0
\end{gathered}
$$

$p+\frac{B^{2}}{8 \pi}-\frac{A}{2 \pi}\left[\rho\left(\frac{\partial v}{\partial t}+u \frac{\partial v}{\partial z}+v^{2}-\Omega^{2}\right)+\frac{J^{2}}{4 \pi}+\frac{B}{8 \pi} \frac{\partial^{2} B}{\partial z^{2}}-\frac{1}{16 \pi}\left(\frac{\partial B}{\partial z}\right)^{2}\right]=p_{e x t}$

where the condition of the constant external pressure, $p_{\text {ext }}$, is imposed. The consideration is restricted by the case of uniform atmospheres without gravity. The set of equations is valid for thin flux tubes of finite diameter. In the limit of the infinitely thin flux tube, the variables of the problem, $B_{\phi}$ and $v_{\phi}$, tend to zero and the equations (3) and (4) for them are dropped out of the set, as well as the terms in the boundary conditions (8), which are proportional to the cross section of the tube. If equations (6) and (7) are written as a flux conservation condition, $B A=$ const, the set of equations (1) $-(8)$ is reduced to the well-known thin-flux-tube approximation of Roberts and Webb (1978). The treatment of linear and nonlinear waves in force-free flux tubes is not possible in the framework of this approximation.

\section{Linear Waves in Force-Free Flux Tubes}

After linearization and introducing the exponential dependence of perturbations on time and coordinate, $B \sim \exp (i \omega t-i k z)$, the equations (1)-(8) are reduced to a dispersion equation,

$$
\begin{aligned}
& \frac{A_{0}}{4 \pi\left(C_{S}^{2}+C_{A}^{2}\right)}\left[\omega^{6}+\left(0.5 \alpha^{2} C_{A}^{2}-4 \Omega_{0}^{2}-k_{z}^{2}\left(2 C_{A}^{2}+C_{S}^{2}\right)\right) \omega^{4}+2 \Omega_{0} \alpha C_{A}^{2} k_{z} \omega^{3}+\right. \\
& +k_{z}^{2}\left[2\left(\alpha^{2} C_{S}^{2} C_{A}^{2}+C_{S}^{2} \Omega_{0}^{2}-0.25 \alpha^{2} C_{A}^{4}\right)+k_{z}^{2} C_{A}^{2}\left(C_{A}^{2}+2 C_{S}^{2}\right)\right] \omega^{2}- \\
& \left.-4 \alpha C_{A}^{2} C_{S}^{2} k_{z}^{3} \Omega_{0} \omega+k_{z}^{4} C_{S}^{2} C_{A}^{2}\left(2 \Omega_{0}^{2}+0.5 \alpha^{2} C_{A}^{2}-k_{z}^{2} C_{A}^{2}\right)\right]- \\
& -\left(\omega^{2}-k_{z}^{2} C_{A}^{2}\right)\left(\omega^{2}-C_{T}^{2} k_{z}^{2}\right)=0
\end{aligned}
$$

where

$$
C_{A}=\frac{B_{z 0}}{\left(4 \pi \rho_{0}\right)^{0.5}}, \quad C_{T}^{2}=\frac{C_{S}^{2} C_{A}^{2}}{C_{S}^{2}+C_{A}^{2}}, \quad \alpha=\frac{2 J_{0}}{B_{z 0}}, \quad C_{S}^{2}=\frac{\gamma p_{0}}{\rho_{0}}
$$


We restrict our consideration to a nonrotating tube $\left(\Omega_{0}=0\right)$ when the dispersion equation (9) reads

$$
\begin{array}{r}
\left(\omega^{2}-C_{T}^{2} k^{2}\right)\left(\omega^{2}-C_{A}^{2} k^{2}\right)-\frac{A_{0}}{4 \pi} \frac{\left(\omega^{2}-C_{A}^{2} k^{2}\right)^{2}\left(\omega^{2}-C_{S}^{2} k^{2}\right)}{C_{A}^{2}+C_{S}^{2}}- \\
-\frac{J_{0}^{2} A_{0} C_{A}^{2}}{2 \pi B_{0 z}^{2}} \frac{\left(\omega^{2}-C_{A}^{2} k^{2}\right)\left(\omega^{2}-C_{S}^{2} k^{2}\right)+2 \omega^{2} k^{2} C_{S}^{2}}{\left(C_{A}^{2}+C_{S}^{2}\right)}=0 .
\end{array}
$$

The first term in the dispersion equation corresponds to the wave propagation in the infinitely thin, untwisted tube $\left(J_{0}=0, A_{0}=0\right)$. The second term shows the influence of the finite cross-section. The third term shows the effect of the twisting. It is simple to show that dispersion appears only due to the second term. The dispersion equation (11) can be rewritten as

$$
\left(C_{A}^{2}+C_{S}^{2}-K C_{A}^{2}\right)\left(\omega^{2}-C_{+}^{2} k^{2}\right)\left(\omega^{2}-C_{-}^{2} k^{2}\right)+\frac{A_{0}}{4 \pi}\left(\omega^{2}-C_{A}^{2} k^{2}\right)^{2}\left(\omega^{2}-C_{S}^{2} k^{2}\right)=0,
$$

where

$$
\begin{gathered}
C_{ \pm}^{2}=C_{A}^{2} \frac{C_{A}^{2}+2 C_{S}^{2}+K\left(C_{S}^{2}-C_{A}^{2}\right) \pm \sqrt{S}}{2\left(C_{A}^{2}+C_{S}^{2}\right)-2 C_{A}^{2} K} \\
S=C_{A}^{4}+2 K\left(3 C_{A}^{2} C_{S}^{2}+4 C_{S}^{4}-C_{A}^{4}\right)+K^{2}\left(C_{S}^{4}-6 C_{S}^{2} C_{A}^{2}+C_{A}^{4}\right) .
\end{gathered}
$$

The parameter $K$ is

$$
K=\frac{J_{0}^{2} A_{0}}{2 \pi B_{0 z}^{2}}=\frac{A_{0} \alpha^{2}}{8 \pi}=\frac{\alpha^{2} R_{0}^{2}}{8},
$$

where $\alpha$ is the well-known parameter of force-free magnetic fields and $R_{0}$ is the radius of the flux tube. If the parameter $K$ tends to zero, the fast speed $C_{+}$ tends to the Alfvén speed $C_{A}$, and the slow speed $C_{-}$tends to the tube speed $C_{T}$. Alfvén torsional and slow magnetosonic sausage waves are modified by the twisting of the tube. The speeds $C_{+}$and $C_{-}$may be considered as the modified Alfvén speed and modified tube speed respectively.

In the case of a weak dispersion, when $\omega \approx C_{ \pm} k$ the dispersion equation (12) reduces to an approximate one

$$
\omega^{2} \approx C_{ \pm}^{2} k^{2}\left(1 \pm \frac{A_{0}}{4 \pi} \frac{\left(C_{ \pm}^{2}-C_{A}^{2}\right)^{2}\left(C_{ \pm}^{2}-C_{S}^{2}\right)}{C_{A}^{2} \sqrt{S}} k^{2}\right),
$$

The case of weak dispersion has a long wavelength limit, when the wavelength is large in comparison with the tube diameter, $k^{2} A_{0} \ll 1$. A weak dispersion limit for torsional Alfvén waves appears also for the case of weakly twisted flux tubes, when $K \ll 1$ and the phase velocity is approximately

$$
C_{+}^{2} \approx C_{A}^{2}\left(1+\frac{K \beta}{2}\right)
$$

In this case the approximate dispersion relation reads

$$
\omega^{2} \approx C_{+}^{2} k^{2}\left(1+\frac{A_{0} K^{2} \beta^{2}(1-\beta)}{16 \pi} k^{2}\right)
$$

which is valid for any values of $k^{2} A$, if the twisting is small enough. 


\section{Discussion}

Eq. (11) cannot be subdivided into the dispersion equations for Alfvén, tube, and other waves. This means that Alfvén, tube, and sausage body wave modes are coupled and pure simple wave modes do not exist. Each of the wave modes is a mixture of Alfvén, tube, and sausage modes. For the case of $K \beta \ll 1$ the wave modes appear to be weakly coupled and they do not differ significantly from the pure Alfvén, tube, or sausage modes in untwisted tubes. But the dispersion equation cannot be subdivided into the dispersion equations for pure MHD modes, as happens in a uniform atmosphere. Slow waves in the forcefree magnetic field are accompanied by torsional disturbances, whereas torsional Alfvén oscillations in force-free magnetic fields are accompanied by pressure, density and temperature fluctuations.

The effect of a coupling of linear wave modes in force-free magnetic fields is essential for the dynamics of solar prominences. There has to be coupling between torsional and pressure oscillations of prominences. This makes possible the excitation of torsional oscillations by arbitrary shaking of the prominence legs. Even pure pressure fluctuations in the legs of prominences produce torsional oscillations as well if there are currents in the prominence. This easily makes the excitation of torsional oscillations of prominences.

The Alfvén torsional waves could be considered as an alternative current. If an alternative current exists along with a constant current, it produces fluctuations of optical emission, which is proportional to product of the strengths of those currents. Consequently, direct observations of alternative currents in prominences are possible.

The general dispersion relation (9) shows that the effect of tube rotation is similar to the influence of tube twisting on linear waves. The wave modes appear to be coupled in the rotating tube. The production of currents in prominences is the result of the twisting, which appears due to rotation of the flux tubes. Consequently, both twisting and rotation couple different modes of prominence oscillations.

The dispersion of Alfvén torsional waves is essential for nonlinear waves (see Zhugzhda and Nakariakov (1998, these proceedings)).

Acknowledgments. The author thanks the Alexander von Humboldt Foundation for support of this research.

\section{References}

Roberts B. and Webb A.R. 1978, Solar Phys., 56, 5

Zhugzhda, Y.D. 1996, Phys. Plasmas, 3, 10 